\title{
Motion Stabilization Control of Electric Vehicle under Snowy Conditions Based on Yaw-Moment Observer
}

\author{
Hiroshi Fujimoto, Takeo Saito and Toshihiko Noguchi \\ Department of Electrical Engineering, Nagaoka University of Technology \\ Nagaoka 940-2188, Japan hfuji@ieee.org
}

\begin{abstract}
In this paper, novel direct yaw moment control and anti-skid control are proposed for electrical vehicles with two in-wheel motors. The proposed controllers are composed of double disturbance observers. The inner-loop observer controls the vehicle traction, and outer-loop observer stabilizes the yawing motion. The advantages of these approaches are 1) the stability robustness for road condition is guaranteed and 2) the proposed controllers require no immeasurable parameters. The experiments demonstrate the performance of these controllers under snowy conditions, and the stability is theoretically analyzed as inertia variation and dead-time system.
\end{abstract}

\section{INTRODUCTION}

Recently, electric vehicles (EVs) including fuel-cell and hybrid vehicles have been developed very rapidly as a solution of energy and environmental problems. From the point of view of control engineering, EVs have much attractive potential. Since electric motors and inverters are utilized in drive system, they have great advantages over internal combustion engine vehicles (ICVs) such as quick and comprehensible torque response and individual control of each wheel [1]. Although several control methods have already been proposed using these merits [2], [3], their controllers depend on some immeasurable parameters such as vehicle velocity, slip angle, or cornering stiffness, which are hard to estimate.

In this paper, novel simple controllers are proposed for EVs with in-wheel motors based on double disturbance observers (DOB). The proposed control algorithm never requires these parameters. First, new anti-skid control is proposed with inner-loop DOB to control longitudinal motion. The stability robustness is theoretically guaranteed by modeling the road condition change as inertia variation with dead-time. Second, as outer-loop lateral controller, advanced direct yaw-moment control (DYC) is proposed based on yaw-moment observer (YMO). This observer can nominalize the yawing dynamics by compensating the unknown nonlinear lateral force and disturbance yaw-moment as lumped disturbance. Finally, the proposed methods are applied to a small EV with two in-wheel motors in rear wheels. Simulations and experiments are performed to show the advantages of proposed methods.

Small EVs are expected to be popular as new transportation system of urban and rural areas as commuter car, second car, delivery service, or elderly persons' vehicle. When these vehicles are driven in cold district, there are

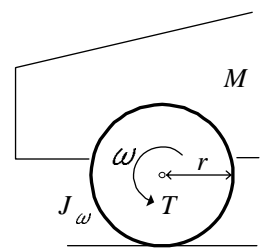

Fig. 1. One-wheel vehicle model.

risks of skid, slip, drift, or spin on snowy or iced road. Thus, the control technologies of braking/traction control and DYC are very important components for safety driving.

\section{ANTI-SKID CONTROL BASED ON ObSERVER}

\section{A. Adhesion and skid motion model}

For anti-skid control, a simple one-wheel model shown in Fig. 1 is utilized. In complete adhesion condition, the longitudinal motion equation can be described as

$$
\left(J_{\omega}+M r^{2}\right) \frac{d \omega}{d t}=T
$$

where $J_{\omega}$ is the wheel inertia, $M$ is the vehicle mass, $r$ is the radius of tire, $T$ is the motor torque, $\omega$ is wheel speed. When the skid phenomenon is occurred on low $\mu$ road, we can regard it as the sudden decrease of the vehicle inertia $M r^{2}$ [1]. In this paper, novel anti-skid control is proposed based on these characteristics.

\section{B. Anti-skid control based on disturbance observer}

In this section, a simple anti-skid control is proposed based on DOB as shown in Fig. 2. Here, $P(s):=$ $\omega(s) / T(s)$ is the controlled plant, $I^{*}$ is the torque current command, $K_{t}$ is the torque constant, $T_{d i s}$ is the disturbance torque, $n$ is the sensor noise, and $\omega$ is the wheel speed.

As stated above, we can model the skid phenomenon caused by road condition as inertia variation. In this paper, the variation is described as the plant uncertainty $\Delta$. Thus, the plant transfer function can be represented as

$$
P(s)=\frac{1}{J s}=\frac{1}{J_{n} s}(1+\Delta),
$$

where $J_{n}:=J_{\omega}+M r^{2}$ is the ideal nominal inertia, $J$ is the real inertia which varies according to the road condition, and $\Delta:=\left(J_{n}-J\right) / J$. In complete adhesion condition, $J$ 


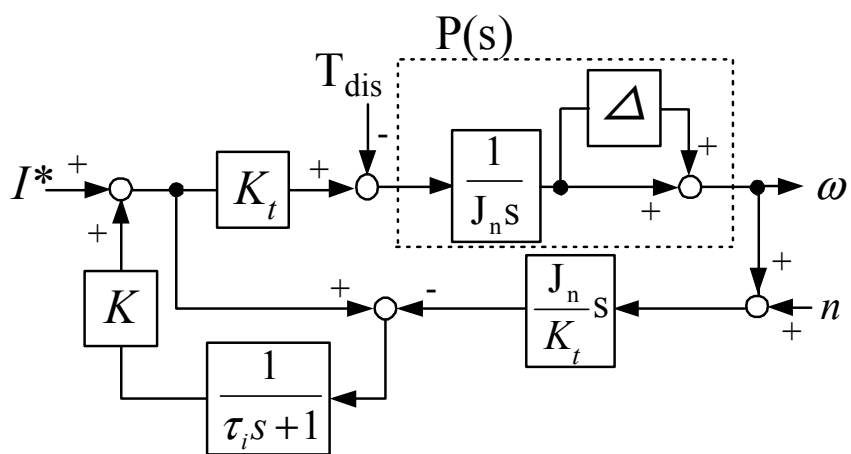

Fig. 2. Block diagram of anti-skid control.

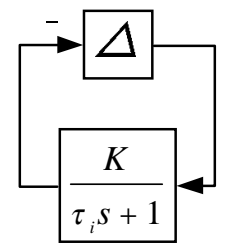

Fig. 3. Equivalent block diagram of Fig. 2.

takes its nominal value $J_{n}$. On the other hand, $J$ becomes $J_{\omega}$ in complete skid condition.

It is well known that the plant can be nominalized by disturbance observer [4]. In this paper, the DOB is applied to anti-skid control. When perfect nominalization is achieved with ideal observer gain $K=1$, the torque response becomes complete adhesion state regardless of $\Delta$ as

$$
\omega(s) \simeq \frac{1}{J_{n} s} T^{*}(s) .
$$

\section{Stability analysis for dead-time system}

If the controlled plant can be described by the simple model as (2), the observer gain can be set to the ideal value $K=1$ and complete adhesion is achieved as (3). However, the real plant has latency caused by 1) wheel speed detection of low resolution encoder, 2) control bandwidth of torque current loop, and 3) the time delay between the motor torque and traction force generation through tire dynamics [5]. Thus, the stability of the proposed system is analyzed by taking the dead-time into account.

The plant with dead-time can be formulated by

$$
P(s)=\frac{1}{J_{s}} e^{-s T_{d}}=\frac{1}{J_{n} s}\{1+\Delta(s)\},
$$

where $T_{d}$ is the equivalent dead-time and the uncertainty can be defined as

$$
\Delta(s)=\frac{J_{n}}{J} e^{-s T_{d}}-1 .
$$

The proposed anti-skid control has to maintain the closed-loop stability against this unmodeled dynamics $\Delta(s)$. Fig. 3 is the equivalent block diagram of Fig. 2 for stability analysis. The stability of the closed-loop system can be analyzed by the open-loop transfer function of Fig.
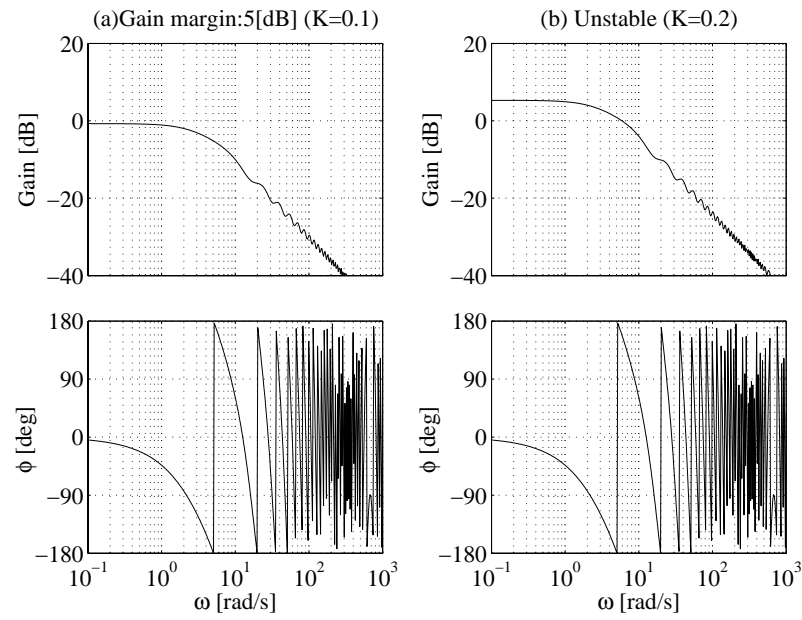

Fig. 4. Frequency response of $Q(s) \Delta(s)$ with $T_{d}=400$ [ms].

2 , which is given by

$$
Q(s) \Delta(s)=\frac{K}{\tau_{i} s+1} \cdot\left(\frac{J_{n}}{J} e^{-s T_{d}}-1\right) .
$$

Here, $Q(s)$ is the complementary sensitivity function composed of low pass filter (LPF) $1 /\left(\tau_{i} s+1\right)$ and observer gain $K$. In this paper, this gain $K$ is determined based on (6) to assure the closed-loop stability.

Fig. 4 is one example when the system has big deadtime $T_{d}=400$ [ms]. The cut-off frequency of LPF is decided to $3[\mathrm{rad} / \mathrm{s}]\left(\tau_{i}=0.33[\mathrm{~s}]\right)$. From the identification experiment on dry road, the nominal inertia is selected as $J_{n}=10.5\left[\mathrm{Nms}^{2}\right]$. By assuming extremely low $\mu$ road, the actual inertia is set to $J=1.0\left[\mathrm{Nms}^{2}\right]$ from the non-load experiment. Although the closed-loop is stable in the case of small $K=0.1$, the system becomes unstable even in $K=0.2$ which is far away from the ideal gain $K=1$. This analysis also means that ICVs cannot achieve high control performance since engines have long time-lag of hundreds millisecond for torque generation.

Moreover, this stability analysis gives good agreement with our former experimental results [6], in which the maximum stable gain was 0.1 . We utilized the commercial inverter which has originally been implemented in our EV by the manufacturer. This inverter had too slow low-pass filter with about 400 [ms] delay for drivers' comfortability. But, this slow filter was nothing but an obstruction for high-speed control. Therefore, in this paper we built new experimental system with handmade inverter in order to minimize the time-delay as possible and to achieve better control performance with higher gain.

Fig. 5 shows the stability analysis of the new experimental system. From our experiments, the dead-time is set to $T_{d}=5[\mathrm{~ms}]$ considering the delay of wheel speed detection by low-resolution encoder $(2[\mathrm{~ms}])$ and time lag between motor torque and traction force generation. The cut-off frequency of LPF is decided to $30[\mathrm{rad} / \mathrm{s}]\left(\tau_{i}=\right.$ $0.033[\mathrm{~s}])$. By removing the big dead-time, the gain can be increased up to $K=0.8$ which corresponds to maximum stable gain in our experimental results of section V-B. 

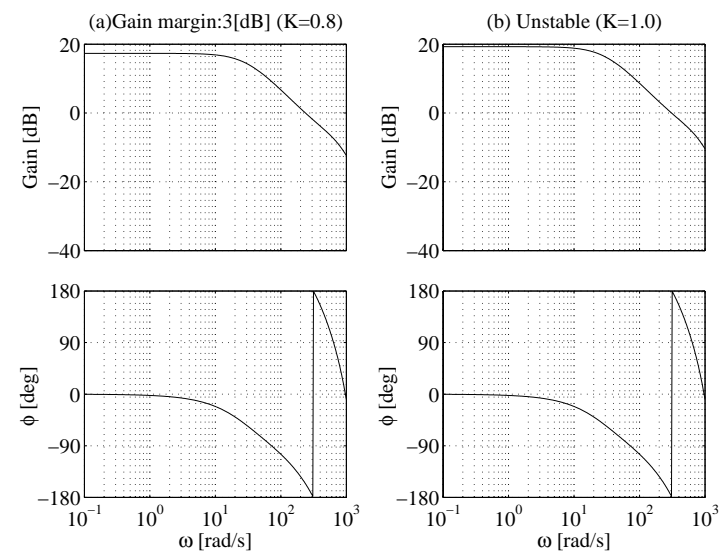

Fig. 5. Frequency response of $Q(s) \Delta(s)$ with $T_{d}=5[\mathrm{~ms}]$.

\section{Direct YAW-MOMENT CONTROL BASED ON OBSERVER}

\section{A. Lateral motion dynamics}

In this section, an advanced DYC is proposed by the use of two in-wheel motors. As shown in Fig. 6, the vehicle is modeled as the equivalent two-wheel model which is sometimes called as half vehicle model. The linearized dynamics of the lateral motion is derived as

$$
\begin{gathered}
M V\left(\frac{d \beta}{d t}+\gamma\right)=2 Y_{f}+2 Y_{r} \\
I \frac{d \gamma}{d t}=N_{z}-N_{t}-N_{d},
\end{gathered}
$$

where $\beta$ is the chassis slip angle, $\gamma$ is the yaw-rate, $I$ is the vehicle inertia, and $V$ is the vehicle velocity which is assumed to be constant. $N_{z}$ is the control moment which is generated by the force difference between the left and right in-wheel motors. $N_{d}$ is the disturbance moment caused by side wind. $N_{t}:=2 l_{r} Y_{r}-2 l_{f} Y_{f}$ is the moment generated by tire and road contact, where $Y_{f}$ and $Y_{r}$ are the lateral force of front and rear wheels, respectively, $l_{f}$ and $l_{r}$ are the distance between the center of mass and wheels.

\section{B. Yaw-moment observer}

It is very difficult to detect the $\beta$ and $V$ directly because we need to measure the vehicle velocity vector. Moreover, the lateral forces $Y_{f}$ and $Y_{r}$ are hard to estimate since they depend on unknown nonlinear parameters. Thus, in this paper novel direct yaw-moment control is proposed based on disturbance observer which compensates these immeasurable terms as lumped disturbance $N_{t}+N_{d}$.

By using the moment $N_{z}$ as control input and yawrate $\gamma$ as measured signal, the disturbance observer can be designed as Fig. 7. This specific disturbance observer is called yaw-moment observer (YMO) in this paper. This YMO can compensate the lumped disturbance and nominalize the system as

$$
\gamma(s) \simeq \frac{1}{I_{n} s} N_{i n}(s) .
$$

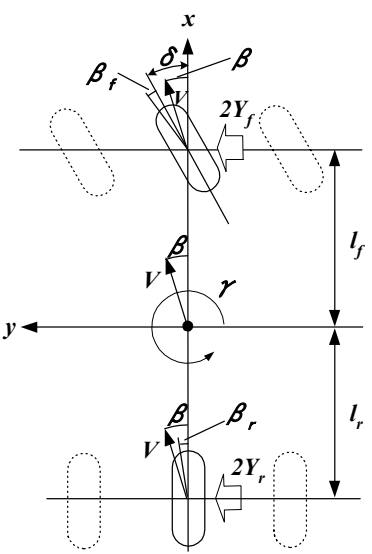

Fig. 6. Equivalent two-wheel vehicle model.

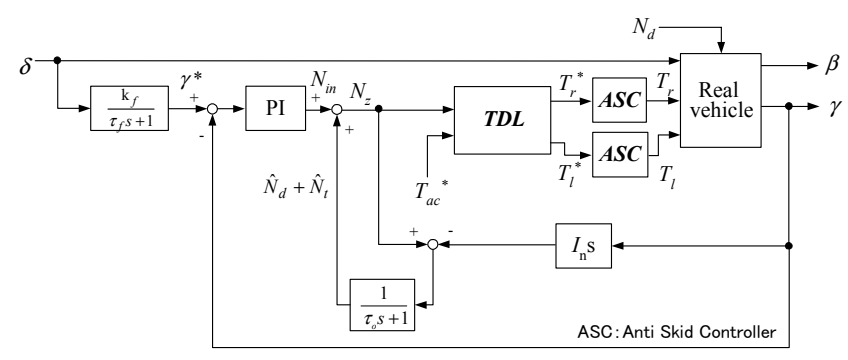

Fig. 7. Block diagram of yaw-moment observer.

Here, we assumed that the arbitrary control moment $N_{z}$ can be generated by the inner-loop anti-skid control proposed in section II.

The control input $N_{z}$ obtained by YMO is distributed to two motors based on the following equations.

$$
\begin{gathered}
N_{z}=l_{p}\left(F_{r}^{*}-F_{l}^{*}\right) \\
T_{a c}^{*}=F_{r}^{*}+F_{l}^{*}
\end{gathered}
$$

$l_{p}$ is the half distance between left and right wheels.

By solving the simultaneous equations (10) and (11), the force command signals to left and right wheels $\left(F_{l}^{*}\right.$ and $F_{r}^{*}$ ) can be determined from $N_{z}$ and accelerator command $T_{a c}^{*}$. Thus, the torque commands to two in-wheel motors can be calculated as

$$
T_{r}^{*}=r F_{r}^{*}, \quad T_{l}^{*}=r F_{l}^{*} .
$$

In Fig. 7, the TDL represents the above torque distribution law.

\section{Feedforward desired model}

In the proposed method of Fig. 7, the desired yaw-rate command $\gamma^{*}$ is obtained by the feedforward model

$$
\gamma^{*}(s)=\frac{k_{f}}{\tau_{f} s+1} \delta(s),
$$

where $\delta$ is the steering angle. In the following simulations, the feedforward parameters are selected as $\tau_{f}=0.05$ [s] and $k_{f}=4$ based on experimental data on dry road. 


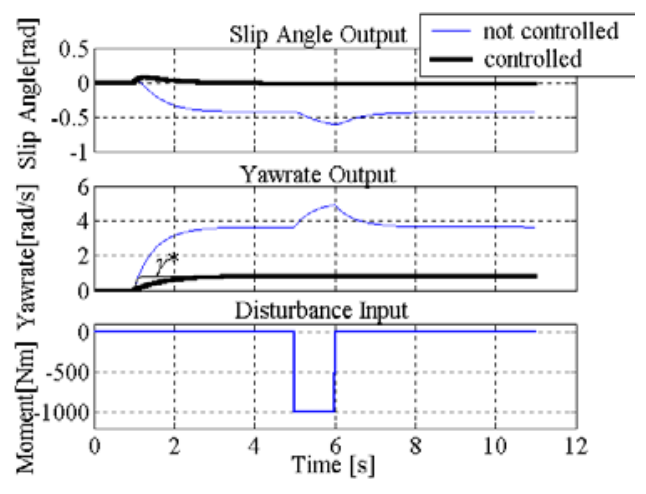

Fig. 8. Simulation results of YMO on dry road.

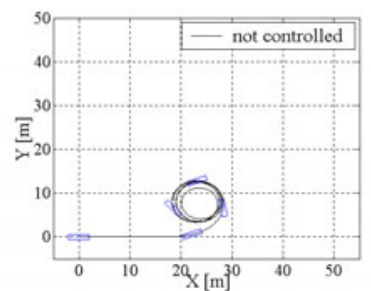

(a) without control

Fig. 9. Trajectories of vehicle on dry road.

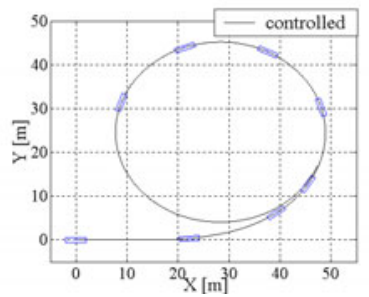

(b) with control

\section{Simulation of YAW-MOMEnt ObSeRVER}

In this section, the proposed DYC with YMO is verified through simulations. It is assumed that the inner-loop antiskid controller can keep the complete adhesion status. Thus, in simulations the plant can be modeled by (7) and (8) with the lateral force given by

$$
\begin{aligned}
Y_{f} & =-C_{f}\left(\beta+\frac{l_{f}}{V} \gamma-\delta\right), \\
Y_{r} & =-C_{r}\left(\beta-\frac{l_{r}}{V} \gamma\right),
\end{aligned}
$$

where $C_{f}$ and $C_{r}$ are the front and rear cornering stiffness, respectively. The simulation parameters are set to $V=60[\mathrm{~km} / \mathrm{h}], M=400[\mathrm{~kg}], l_{f}=0.689[\mathrm{~m}]$, and $l_{r}=0.591[\mathrm{~m}]$. As the steering signal $\delta$, the step-type function with amplitude $0.2[\mathrm{rad}]$ is injected at $t=1$ [s]. The disturbance yaw-moment $N_{z}$ is added in $t=5 \sim 6$ [s].

Fig. 8 shows the simulation results on dry road condition with $C_{f}=C_{r}=9500[\mathrm{~N} / \mathrm{rad}]$. The yaw-rate $\gamma$ follows the desired command $\gamma^{*}$ very well and the disturbance is also well rejected by the proposed YMO. The vehicle trajectories are shown in Fig. 9. In the case without control, the vehicle becomes oversteer situation and the trajectory is varied by the disturbance. On the other hand, the ideal stable trajectory is obtained by the proposed controller.

In Fig. 8, the YMO is checked in much severer conditions. The cornering stiffness is assumed to decrease 50 [\%] considering snowy road and the vehicle velocity is set to $V=80[\mathrm{~km} / \mathrm{h}]$. In the case without control, the vehicle motion becomes unstable. However, the yaw-rate is still stable by the proposed controller.

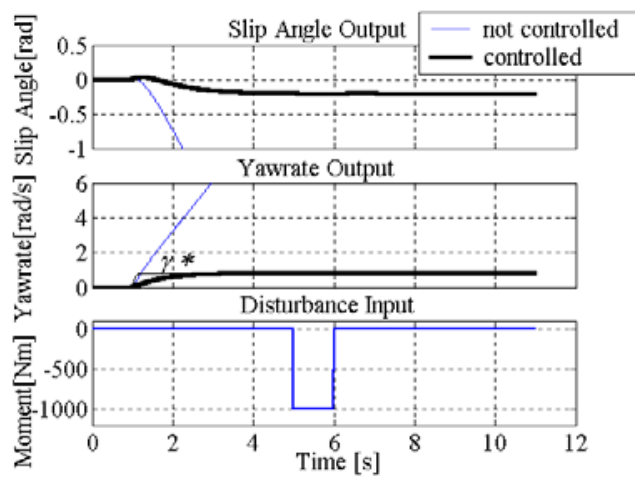

Fig. 10. Simulation results of YMO on snow road.

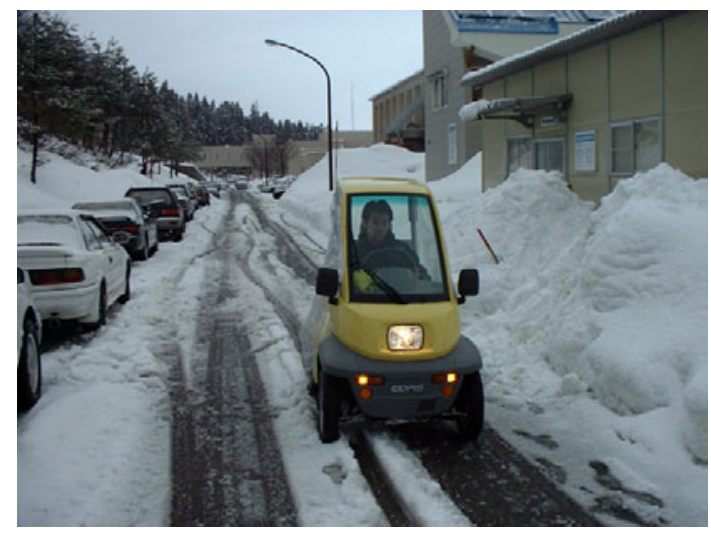

Fig. 11. Photograph of experimental setup.

\section{EXPERIMENTAL RESULTS}

\section{A. Experimental setup}

To verify the proposed control algorithm, an experimental setup was constructed based on a commercial EV "COMS" made by Araco corporation, which has two in-wheel motors (Fig. 11). We built IGBT inverters by ourselves to minimize the time-delay in control loop. The switching frequency of inverters is 10 [kHz].

As shown in Fig. 12, the controllers are implemented in a PC and feedback signals such as yaw-rate sensor and motor current are sampled by A/D board through PCI Buss interface. The command signals to two motors are transmitted to inverters through D/A converter and modulation circuits. The proposed control system has triple-loop structure composed of 1) current control as first innerloop, 2) anti-skid control as second inner-loop, and 3) yaw-moment observer as outer-loop system. These three controllers can be calculated by one PC with RT-Linux. The control period is $100[\mu \mathrm{s}]$ which is synchronous with the career signal of inverters.

The wheel speed is detected by encoder pulse which is obtained at every 60 electrical degree. The resolution is about 225 pulse per revolution of the tire. The noise problem is caused by the quantization with this low resolution encoder. Thus, the wheel speed is calculated with the moving average during 2 [ms]. 


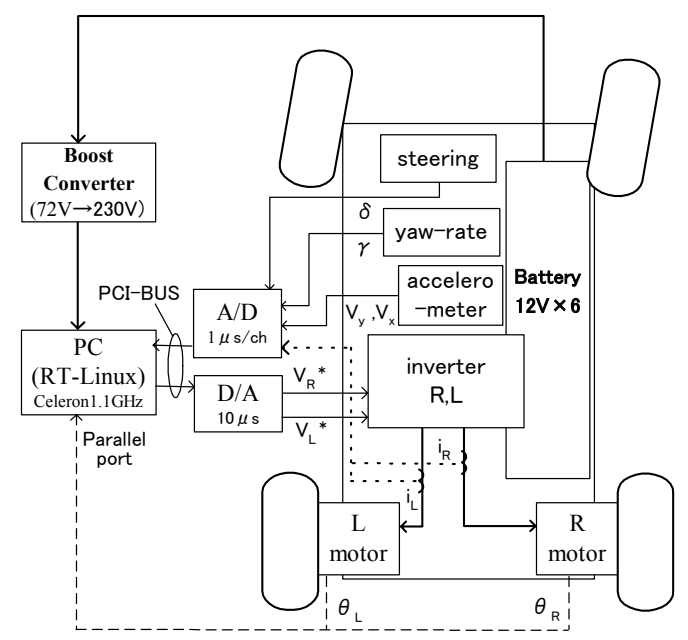

Fig. 12. Configuration of experimental setup.

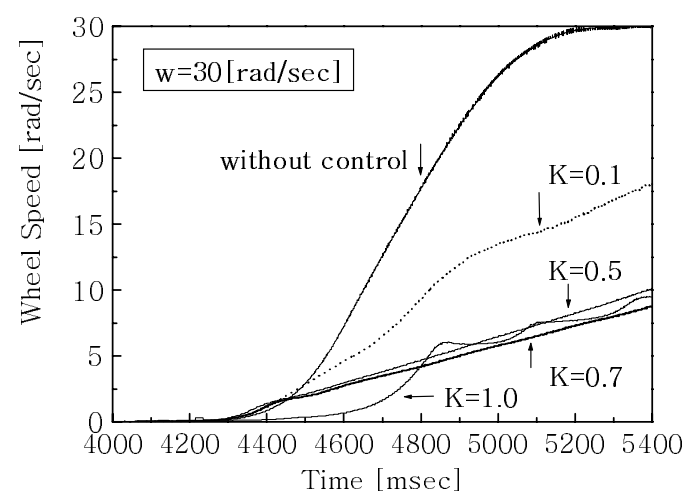

Fig. 13. Experimental results of anti-skid control.

\section{B. Experiments on anti-skid control}

The experiments of anti-skid control (Fig. 2) were performed on an asphalt-paved road covered with snow. The command signal $I^{*}$ is 45 [A] step-type function which starts from $t=4000[\mathrm{~ms}]$. The experimental results of wheel speed are shown in Fig. 13 and Fig. 14 with various $K$ and $\omega_{i}\left(=1 / \tau_{i}\right)$. In Fig. 13, the cut-off frequency is fixed to $\omega_{i}=30[\mathrm{rad} / \mathrm{s}]$ and the observer gain is tuned in $K=0.0 \sim 1.0$. In the case without control, the wheel speed increases too rapidly, which means the wheel-spin is occurred. On the other hand, the proposed controller prevents the wheel-spin and the wheel speed can accelerate mildly. The control performance gets better by higher gain $K$. However, the unstable oscillation is occurred by the ideal gain $K=1$. The reason can be explained by the stability analysis of Fig. 5. The maximum stable gain obtained in experiments $(K=0.8)$ gives good agreement with the value that has been obtained by the theoretical calculation in section II-C. In Fig. 14 the cut-off frequency is tuned at $\omega_{i}=3,30$ and $40[\mathrm{rad} / \mathrm{s}]$ under the fixed observer gain $K=0.8$. The best value is $\omega_{i}=30[\mathrm{rad} / \mathrm{s}$ ] in these experiments.

Fig. 15 shows the vehicle velocity which is calculated by the digital integration of accelerometer signal with 100

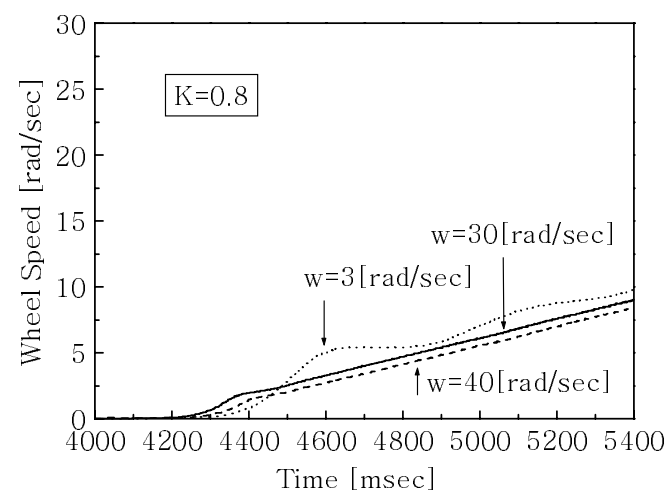

Fig. 14. Improvement by cut-off frequency.

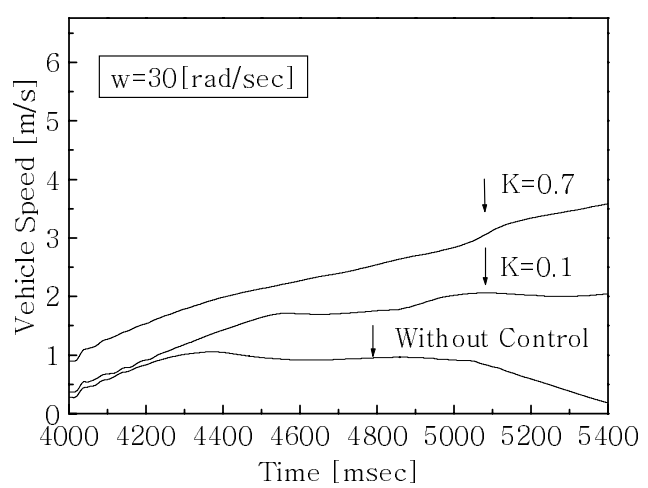

Fig. 15. Vehicle velocity by accelerometer.

[ $\mu \mathrm{s}]$ sampling period. The cut-off frequency is fixed at $\omega_{i}=$ $30[\mathrm{rad} / \mathrm{s}]$ and the observer gain is changed at $K=0.0,0.1$, and 0.7 . The offset at initial time $t=4000[\mathrm{~ms}]$ is caused by the integral of inverter switching noise from the starting time $t=0$. However, the gradient is meaningful since the inverter noise is filtered by the integral action. In case of $K=0.7$, we find that the vehicle keeps adhesion and the velocity increases with constant acceleration because the wheel speed is well controlled as shown in Fig. 13. On the other hand in lower gain $K=0$ and 0.1 , the vehicle loses the adhesion and the velocity decreases by the wheel-spin phenomenon.

In Fig. 16, the vehicle velocity is compared with wheel speed at $K=0.7, \omega=30[\mathrm{rad} / \mathrm{s}]$. The upper line is obtained from the integration of accelerometer signal. The lower line is calculated from the encoder signal. Although the upper line has offset, the gradient of these two line is almost same. This means that the proposed controller keeps the adhesion and prevents the wheel-spin phenomenon.

\section{Experiments on yaw-moment observer}

Next, the control performance of proposed YMO is verified against the yaw-moment disturbance. After the vehicle has been accelerated by the constant torque 75 $[\mathrm{Nm}]$, the velocity is controlled at $25[\mathrm{~km} / \mathrm{h}]$. During it runs straight at this constant speed, the driver gives the disturbance by steering.

Because the desired yaw-rate is intentionally set to $\gamma^{*}=$ 


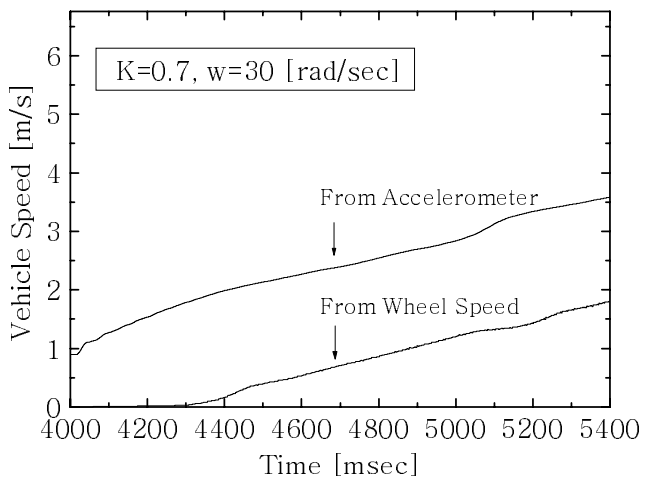

Fig. 16. Wheel speed and accelerometer output.

0 in the controller, the steering motion can be regarded as the yaw-moment disturbance $N_{d}$. In order to make the comparison fair, almost same disturbance is generated by checking the output of steering angle sensor although the steering is manipulated by a human driver. The parameters of PI controller are $K_{p}=200$ and $K_{i}=5$.

Fig. 17 shows the experimental results on dry asphalt road. The upper line is the output of steering angle sensor and the lower one is that of the yaw-rate sensor. We find that the proposed controller improves the yaw-rate 33 [\%]. The sinusoidal waveform of steering angle means that 1) the first half period is the disturbance made by the driver and 2) the second half period is the adjust steering of driver in order to run straight after the direction is changed by the disturbance. It is shown that the adjust steering becomes smaller by the proposed controller.

The experimental results on the snowy road are shown in Fig. 18. The reason of slight yaw-rate oscillation in $t=$ $2 \sim 5[\mathrm{~s}]$ is that the lateral force decreases by the little skid in acceleration time. During the yaw-rate disturbance is injected in $t=8 \sim 9[\mathrm{~s}]$, the proposed controller makes the yaw-rate 25 [\%] smaller than the response without control. Although the spin phenomenon is occurred at $t=11[\mathrm{~s}]$ in the case without control, the proposed controller can prevent the dangerous situation.

The disturbance rejection performance of experiments is less than that of simulations. The reason is that the cut-off frequency of YMO becomes very law $\omega_{o}\left(=1 / \tau_{o}\right)=0.3$ $[\mathrm{rad} / \mathrm{s}]$ because of some technical problems such as the large noise of handmade inverter. However, we expect that it will be possible to choose the cut-off frequency over $\omega_{o}=3[\mathrm{rad} / \mathrm{s}]$ if these problems are removed after the improvement of our experimental setup. This will be one of our future works.

\section{CONCLUSION}

This paper proposed simple and high performance controllers for electric vehicles based on double disturbance observers. The inner-loop observer can achieve anti-skid control by modeling the road change as plant variation. The stability of closed-loop system is theoretically analyzed as dead-time system and its validity is confirmed by experiments.

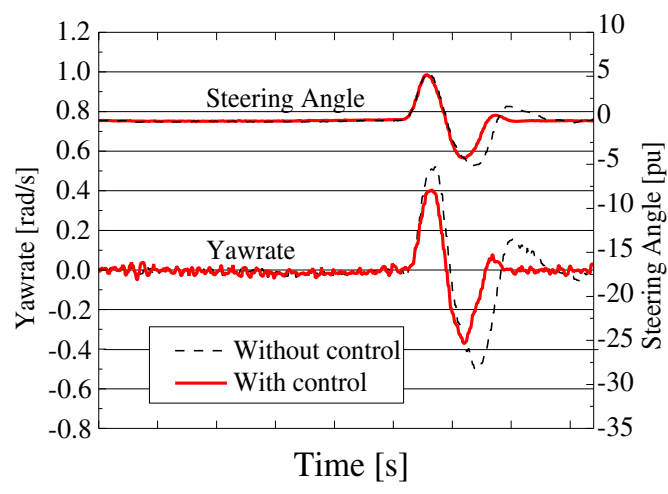

Fig. 17. Experimental results of YMO on dry road.

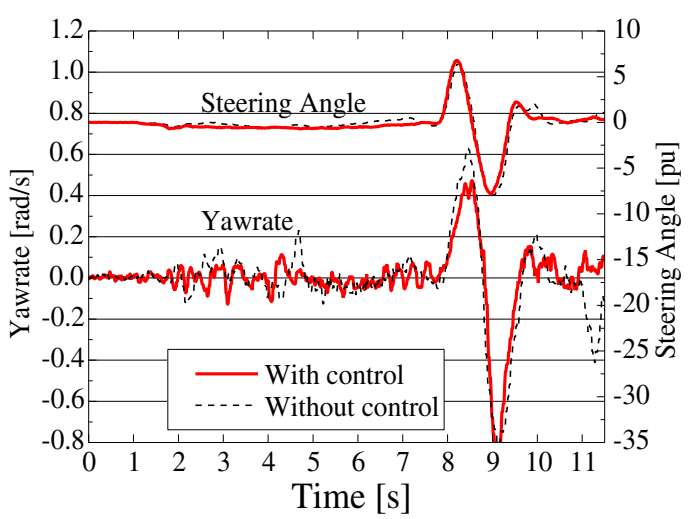

Fig. 18. Experimental results of YMO on snowy road.

The outer-loop yaw-moment observer can estimate and compensate the yaw-moment disturbance and the unknown nonlinear lateral force as lumped disturbance. Thus, the proposed controller does not need any immeasurable parameters such as slip angle or cornering stiffness. The experiments was carried out on the dry and snowy roads. The disturbance yaw-moment was attenuated maximum 33 [\%] by this controller. The future work will be the improvement of experimental setup to achieve higher cutoff frequency.

\section{REFERENCES}

[1] Y. Hori, S. Sakai, H. Sado and T. Uchida: "Motion control of electric vehicle utilizing fast torque response of electric motor", 14th IFAC World Congress, Vol. P, pp. 491-496 (1999).

[2] S. Sakai, H. Sado and Y. Hori: "Motion control in an electric vehicle with 4 independently driven in-wheel motors", IEEE/ASME Trans. Mechatronics, 4, 1, pp. 9-16 (1999).

[3] M. Shino, Y. Wang and M. Nagai: "Motion control of electric vehicles considering vehicle stability", AVEC 2000, pp. 645-652 (2000).

[4] K. Ohnishi, M. Shibata and T. Murakami: "Motion control for advanced mechatronics", IEEE/ASME Trans. Mechatronics, 1, 1, pp. 56-67 (1996)

[5] T. Umeno, K. Asano, H. Ohashi, M. Yonetani, T. Naitou and T. Taguchi: "Observer based estimation of parameter variations and its application to tire pressure diagnosis", Control Engineering Practice, 9, 6, pp. 639-645 (2001).

[6] T. Saito, H. Fujimoto and T. Noguchi: "Yaw-moment stabilization control of small electric vehicle", IEE of Japan Technical Meeting Record, No. IIC-02-53, pp. 83-88 (2002). (in Japanese). 